\title{
Метод трехкомпонентной зонной плавки: моделирование концентрационного распределения компонентов в монокристаллах твердых растворов $\mathrm{Ge}-\mathrm{Si}$
}

\author{
(C) 3.А. Агамалиев ${ }^{1,2}$ \\ ${ }^{1}$ Бакинский государственный университет, \\ Az-1148, Баку, Азербайджан \\ ${ }^{2}$ Институт физики Национальной академии наук Азербайджана, \\ Az-1143, Баку, Азербайджан \\ E-mail: zohrabagamaliyev@bsu.edu.az \\ Поступила в Редакцию 14 октября 2020 г. \\ В окончательной редакции 19 октября 2020 г. \\ Принята к публикации 19 октября 2020 г.
}

Дана концепция и теоретическая основа метода трехкомпонентной зонной плавки для выращивания монокристаллов полупроводниковых твердых растворов с использованием затравок из составных компонентов. В пфанновском приближении решена задача аксиального концентрационного распределения компонентов в монокристаллах $\mathrm{Ge}-\mathrm{Si}$, выращенных при различных значениях операционных параметров, таких как длина расплавленной зоны и состав исходных макрооднородных стержней твердых растворов. Анализ полученных результатов определяет потенциал метода и оптимальные условия для выращивания монокристаллов с заданными однородным и градиентным составами во всем непрерывном ряду твердых растворов $\mathrm{Ge}-\mathrm{Si}$. Показана перспективность метода трехкомпонентной зонной плавки для выращивания монокристаллов полупроводниковых твердых растворов.

Ключевые слова: Полупроводниковые твердые растворы, распределение компонентов, материал затравки.

DOI: 10.21883/FTP.2021.02.50512.9535

\section{1. Введение}

Научная значимость и перспективность масштабного практического использования полупроводниковых твердых растворов ставят технологические задачи по выращиванию их объемных монокристаллов в разряд приоритетных. Анализ литературных данных по данной проблеме показывает, что задачу по обеспечению лабораторных исследований монокристаллами полупроводниковых твердых растворов в принципе можно считать решенной, несмотря на все еще имеющиеся сложности. Актуальной остается проблема по получению монокристаллов твердых растворов в промышленных масштабах [1]. Это обстоятельство мотивирует работы, направленные на совершенствование и модификацию существующих методов, а также на создание новых методов выращивания монокристаллов твердых растворов с заданными составом и качеством [2-20].

Проблема выращивания объемных монокристаллов полупроводниковых твердых растворов требует решения следующих основных задач [1,17].

- Обеспечить заданное однородное или градиентное аксиальное распределение компонентов в кристаллах.

- Обеспечить наличие соответствующих затравок для инициации роста монокристаллов твердых растворов из расплавов с различным составом.

- Определить оптимальные технологические условия и режимы кристаллизации расплава, обеспечивающие монокристалличность твердых растворов.
В настоящей работе представлены концепция и теоретическая основа выращивания монокристаллов полупроводниковых твердых растворов с заданным составом новым методом трехкомпонентной зонной плавки, а также результаты математического моделирования концентрационного распределения компонентов в кристаллах $\mathrm{Ge}-\mathrm{Si}$, выращенных этим методом при различных значениях операционных параметров.

Цель работы - определение потенциала и перспектив предложенного метода для выращивания монокристаллов полупроводниковых твердых растворов с заданным однородным и (или) градиентным составами.

Для удобства и большей конкретности, в качестве примера рассмотрим классическую систему твердых растворов кремний-германий, составные компоненты которой растворяются друг в друге при любых соотношениях, как в жидком, так и в твердом состояниях $[1,21]$.

\section{2. Концепция и теоретическое обоснование}

На рис. 1 представлены схематические диаграммы выращивания кристаллов $\mathrm{Ge}-\mathrm{Si}$ методом трехкомпонентной зонной плавки с использованием затравок из $\mathrm{Ge}$ (рис. $1, a)$ и $\mathrm{Si}$ (рис. 1,b). В обоих случаях в тигель цилиндрической формы закладываются предварительно изготовленные три стержня (компонента), с соответствующими составами и размерами. В первом случае 




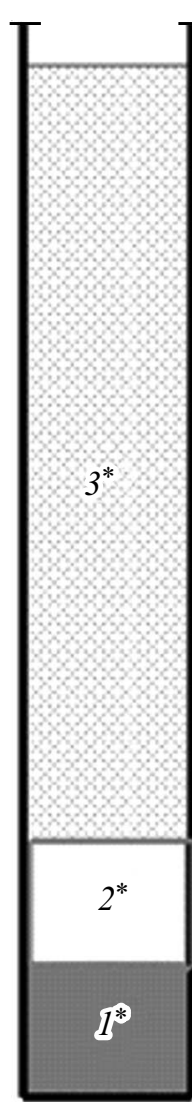

A

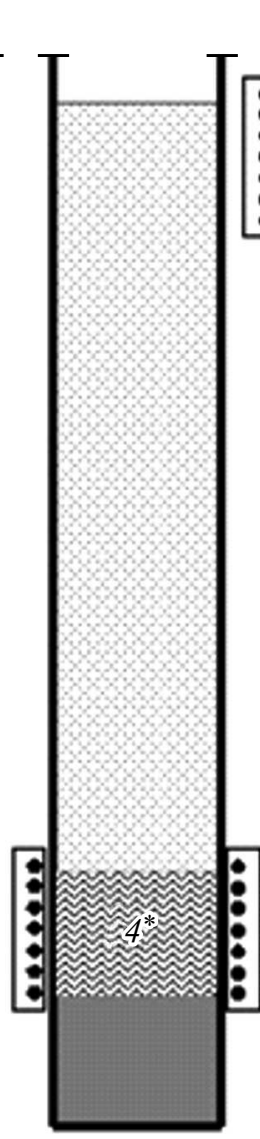

$\mathrm{B}$

$b$

Рис. 1. Схематические диаграммы выращивания кристаллов $\mathrm{Ge}-\mathrm{Si}$ методом трехкомпонентной зонной плавки с использованием затравок из $\mathrm{Ge}(a)$ и $\mathrm{Si}(\mathrm{b}): \mathrm{A}$ - порядок загрузки тигля стержнями исходных материалов; В - предстартовый момент кристаллизации первичной расплавленной зоны длиной $Z$; $\mathrm{C}-$ момент образования финальной расплавленной зоны длиной $Z$ : $1,1^{*}$ - затравки из $\mathrm{Ge}$ и $\mathrm{Si}$ соответственно; 2,2*- $\mathrm{Ge}_{1-x} \mathrm{Si}_{x^{*}}$ стержни соответственно; 3, 3* - стержни $\mathrm{Ge}_{1-x} \mathrm{Si}_{x} ; 4,4^{*}-$ стартовые расплавленные зоны из $\mathrm{Ge}$ и $\mathrm{Ge}_{1-x^{*}} \mathrm{Si}_{x^{*}}$ соответственно; 5, $5^{*}$ - растущие монокристаллы $\mathrm{Ge}-\mathrm{Si} ; 6,6^{*}-$ финальные расплавленные зоны длиной $Z$.

(рис. $1, a-\mathrm{A})$, в тигле над монокристаллической затравкой из Ge последовательно размещаются стержень из поликристаллического германия и предварительно изготовленный макрооднородный подпитывающий стержень $\mathrm{Ge}_{1-x} \mathrm{Si}_{x}$ с заданным $x$. В условиях вакуума проводится расплавление стержня из $\mathrm{Ge}$, расположенного непосредственно над затравкой (рис. 1, $a-$ В). В предстартовый момент начала роста кристалла температура на границах расплава с затравкой и верхним слитком равна температуре плавления германия. Рост кристалла происходит на затравке с момента включения механизма перемещения нагревателя относительно тигля и продолжается до полной перекристаллизации подпитывающего стержня $\mathrm{Ge}_{1-x} \mathrm{Si}_{x}$. До момента образование конечной расплавленной зоны (рис. $1, a-\mathrm{C}$ ) длина зоны поддерживается постоянной и равной $Z$. В отличие от традиционного метода зонной плавки, здесь начальная расплавленная зона состоит из чистого германия. Это обстоятельство решает проблему затравки, необходимой для получения монокристаллов $\mathrm{Ge}-\mathrm{Si}$ с различным составом путем использования германиевой затравки.

Для выращивания монокристаллов $\mathrm{Ge}-\mathrm{Si}$, с использованием затравки из $\mathrm{Si}$ (рис. $1, b-\mathrm{A}$ ), в тигель над затравкой закладываются макрооднородные стержни с составами $\mathrm{Ge}_{1-x} \mathrm{Si}_{x *}$ и $\mathrm{Ge}_{1-x} \mathrm{Si}_{x}$. Стержень, размещенный непосредственно над затравкой, служит для создания стартовой расплавленной зоны длиной $Z$ (рис. $1, b-\mathrm{B}$ ). Состав его $x^{*}$ определяем из диаграммы равновесного фазового состояния системы $\mathrm{Ge}-\mathrm{Si}$, который равен составу расплава, находящегося в фазовом равновесии с верхним подпитывающим стержнем с заданным $x$. В предстартовый момент кристаллизации расплава температура на границах расплава с затравкой и верхним стержнем равна температуре ликвидуса твердого раствора $\mathrm{Ge}_{1-x *} \mathrm{Si}_{x^{*}}$. Рост монокристалла начинается на затравке с момента включения механизма перемещения нагревателя относительно тигля. Как и в случае с использованием затравки и $\mathrm{Ge}$, значение $Z$ в процессе перекристаллизации верхнего стержня поддерживается 
неизменным вплоть до образования финальной расплавленной зоны (рис. $1, b-\mathrm{C}$ ).

Заметим, что возможность использования затравки из более тугоплавкого компонента для выращивания монокристаллов твердых растворов была показана в работе [22]. Автором этой работы методом Чохральского, с использованием затравки из $\mathrm{Si}$ были выращены монокристаллы $\mathrm{Ge}-\mathrm{Si}$ переменного состава из расплавов с различным содержанием Si.

Задачу концентрационного распределения компонентов вдоль кристалла $\mathrm{Ge}-\mathrm{Si}$, выращенного методом трехкомпонентной зонной плавки, решали в пфанновском приближении при выполнении следующих стандартных условий [21]: фронт кристаллизации плоский; скорости диффузии атомов $\mathrm{Si}$ и $\mathrm{Ge}$ в расплаве обеспечивают его однородность по всему объему (полностью размешанный расплав); на фронте кристаллизации существует равновесие между жидкой и твердой фазами и коэффициенты сегрегации компонентов расплава изменяются с его составом в соответствии с диаграммой равновесного фазового состояния системы $\mathrm{Ge}-\mathrm{Si}$; термическое расширение или сжатие материала при фазовых переходах пренебрежимо мало.

Отметим, что вопросы по моделированию концентрационного распределения компонентов в кристаллах $\mathrm{Ge}-\mathrm{Si}$, выращенных из расплава рядом консервативных и неконсервативных методов, были решены в пфанновском приближении в работах [5-9,23]. Результаты их показали хорошее согласие с экспериментальными данными.

Введем следующие обозначения: $V_{m}^{0}$ и $V_{m}$ - объемы расплавленной зоны в начальный и текущий моменты; $C$ - общая концентрационная доля атомов второго компонента $(\mathrm{Si})$ в расплаве; $C_{m}^{0}-$ концентрационная доля $\mathrm{Si}$ в расплавленной зоне в начальный момент; $C_{c}, C_{i}, C_{m}-$ концентрационные доли атомов $\mathrm{Si}$ в растущем кристалле, подпитывающем стержне (рис. 1, $a$ и $b)$ и в расплавленной зоне соответственно; $V_{c}$ объем расплава, кристаллизирующийся в единицу времени; $V_{i}$ - объем подпитывающего стержня, расплавляющийся в единицу времени; $K=C_{c} / C_{m}$ - равновесный коэффициент сегрегации $\mathrm{Si} ; L-$ общая длина исходных стержней над затравкой; $Z$ —длина расплавленной зоны; $l$ - длина перекристаллизированной части материала от затравки.

В принятых выше обозначениях имеем

$$
C_{m}=\frac{C}{V_{m}}, \quad \frac{d C_{m}}{d t}=\frac{\dot{C} V_{m}-\dot{V}_{m} C}{V_{m}^{2}} \text { и } V_{m}=V_{m}^{0}-\left(V_{c}-V_{i}\right) t
$$

По условию задачи, как и в случае традиционного метода зонной плавки, считаем: в процессе всего технологического цикла скорость кристаллизации расплава $\left(V_{c}\right)$ не зависит от времени; до момента образования конечной расплавленной зоны значения параметров $Z$ и $V_{i}$ остаются неизменными. В этом случае, на участке тигля длиной $L-Z$ от затравки, при использовании германиевой затравки (рис. 1,a) справедливы следующие уравнения:

$$
V_{m}=V_{m}^{0}, \quad C_{m}^{0}=0, \quad V_{i}=V_{c} \quad \text { и } \dot{C}=V_{i} C_{i}-V_{c} C_{m} K .
$$

С учетом (2) из уравнений (1) после ряда преобразований, разделения переменных и интегрирования имеем

$$
\int_{0}^{C_{m}} \frac{d C_{m}}{C_{i}-C_{m} K}=\frac{V_{c} t}{V_{m}^{0}}=\frac{1}{Z}
$$

Для случая с кремниевой затравкой (рис. $1, b$ ), до момента образования конечной расплавленной зоны

$V_{m}=V_{m}^{0}, \quad V_{i}=V_{c}, \quad \dot{C}=0, C_{m}=C_{m}^{0} \quad$ и $C_{c}=C_{m}^{0} K^{*}=C_{i}$.

Здесь $K^{*}$ - коэффициент сегрегации $\mathrm{Si}$, соответствующий стартовому составу расплава с $C_{m}^{0}=x^{*}$. Согласно (4), с момента начала кристаллизации до образования конечной расплавленной зоны имеет место рост однородного кристалла с составом, равным таковому исходного подпитывающего слитка.

С момента образования конечной расплавленной зоны, при использовании как $\mathrm{Ge}-$, так и $\mathrm{Si}$-затравок имеем

$$
V_{m}=V_{m}^{0}-V_{c} t, \quad \dot{V}_{m}=-V_{c}, \quad \dot{C}=-V_{c} C_{m} K .
$$

С учетом (5) из (1) после преобразований, разделения переменных и интегрирования получим

$$
\int_{C_{m f}}^{C_{m}} \frac{d C_{m}}{C_{m f}^{0}-C_{m} k}=\ln \frac{V_{m}^{0}}{V_{m}^{0}-V_{c} t}
$$

Здесь $C_{m f}^{0}$ - концентрационная доля $\mathrm{Si}$ в расплаве в момент образования финальной расплавленной зоны длиной Z. Обозначив длину и долю кристаллизированной части расплава конечной зоны $\left(V_{c} t / V_{m}^{0}\right)$ в момент $t$ соответственно символами $l^{*}$ и $\gamma$, запишем (6) в следующем виде:

$$
\gamma \equiv \frac{l^{*}}{Z}=1-\exp \left[-\int_{C_{m}}^{C_{m f}^{0}} \frac{d C_{m}}{C_{m} K-C_{m}}\right] .
$$

Определение $l / Z$ и $\gamma$ как функцию $C_{m}$, равно как и $C_{c}=K C_{m}$, вдоль всей длины материала, подвергнутого зонной перекристаллизации, требует решения интегралов в уравнениях (3) и (7), в которые входит коэффициент сегрегации $K$, зависящий от $C_{m}$.

В системе $\mathrm{Ge}-\mathrm{Si}$ значение $K$ изменяется с составом расплава сложным образом и в широких пределах $[1,24]$. Это обстоятельство приводит к необходимости вычисления интегралов в (3) и (7) численным методом путем определения сопряженных значений $K=C_{c} / C_{m}$ в соответствующих интервалах изменения $C_{m}$ по данным 
диаграммы равновесного фазового состояния системы. В работе [24] было показано, что во всем интервале изменения $C_{m}$, температуры кривых ликвидуса $T_{l}\left(C_{m}\right)$ и солидуса $T_{s}\left(C_{m}\right)$ системы $\mathrm{Ge}-\mathrm{Si}$ достаточно хорошо описываются следующими полиномами пятой степени:

$$
\begin{aligned}
T_{l}\left(C_{m}\right)= & 938.72^{\circ} \mathrm{C}+p_{1} C_{m}+p_{2}\left(C_{m}\right)^{2}+p_{3}\left(C_{m}\right)^{3} \\
& +p_{4}\left(C_{m}\right)^{4}+p_{5}\left(C_{m}\right)^{5}, \\
T_{s}\left(C_{m}\right)= & 938.72^{\circ} \mathrm{C}+q_{1} C_{m}+q_{2}\left(C_{m}\right)^{2}+q_{3}\left(C_{m}\right)^{3} \\
& +q_{4}\left(C_{m}\right)^{4}+q_{5}\left(C_{m}\right)^{5},
\end{aligned}
$$

Здесь $938.72^{\circ} \mathrm{C}$ - температура плавления $\mathrm{Ge}, p_{1}=$ $=1.523764 \cdot 10^{3}, p_{2}=-3.893151 \cdot 10^{3}, p_{3}=6.84677 \cdot 10^{3}$, $p_{4}=-6.011559 \cdot 10^{3}, \quad p_{5}=2.007591 \cdot 10^{3}, \quad q_{1}=$ $=2.440722 \cdot 10^{2}, q_{2}=3.29571 \cdot 10^{2}, q_{3}=-8.419889 \cdot 10^{2}$, $q_{4}=1.543233 \cdot 10^{3}, \quad q_{5}=8.023673 \cdot 10^{2}$. При этом абсолютная ошибка в численных значениях $T_{l}\left(C_{m}\right)$ и $T_{s}\left(C_{m}\right)$ во всем температурном интервале не превышает 1.69 и $0.39^{\circ} \mathrm{C}$ соответственно. Учитывая (8) и (9), значения $K=C_{c} / C_{m}$ в настоящей работе определяли следующим образом. Задавая последовательно значения $C_{m}$ в интервалах указанных в интегралах уравнений (3) и (7), из (8) определяли соответствующие температуры ликвидуса. Затем, приравняв конкретные значения $T_{l}\left(C_{m}\right) \quad$ к $T_{s}\left(C_{m}\right)$, из уравнения (9) определяли сопряженные с каждым значением $C_{m}$ значения $C_{c}$ и $K$ при температурах фазового равновесия системы.

\section{3. Моделирование аксиального концентрационного распределения компонентов в монокристаллах $\mathrm{Ge}-\mathrm{Si}$}

На рис. 2 представлены характерные кривые концентрационного распределения кремния по длине кристаллов $\mathrm{Ge}-\mathrm{Si}$, выращенных методом трехкомпонентной зонной плавки с использованием затравки из $\mathrm{Ge}$ и $\mathrm{Si}$. Расчеты проведены для пяти различных значений $C_{i}$ исходного подпитывающего стержня $\mathrm{Ge}-\mathrm{Si}$ при длине расплавленной зоны $Z=0.1 L$. Кривые, соответствую-

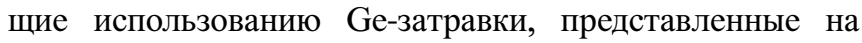
рис. 2, $a$, рассчитаны из уравнений (3) и (7) и соотношения $C_{c}=C_{m} K$. Как видно из рис. $2, a$, в начальной части всех кристаллов аксиальная концентрация $\mathrm{Si}$ растет с довольно большой скоростью, затем, по мере дальнейшего роста кристалла, достигает насыщения, при котором $C_{c} \approx C_{i}$. Протяженность начального участка с растущей концентрацией кремния зависит от состава исходного слитка, значения $K$ и скорости его изменения с составом расплавленной зоны. Обращается внимание на достаточно быстрый переход кривых 1-3 в область плато, свидетельствующий о росте однородных кристаллов $\mathrm{Ge}-\mathrm{Si}$ на достаточно большом протяжении.
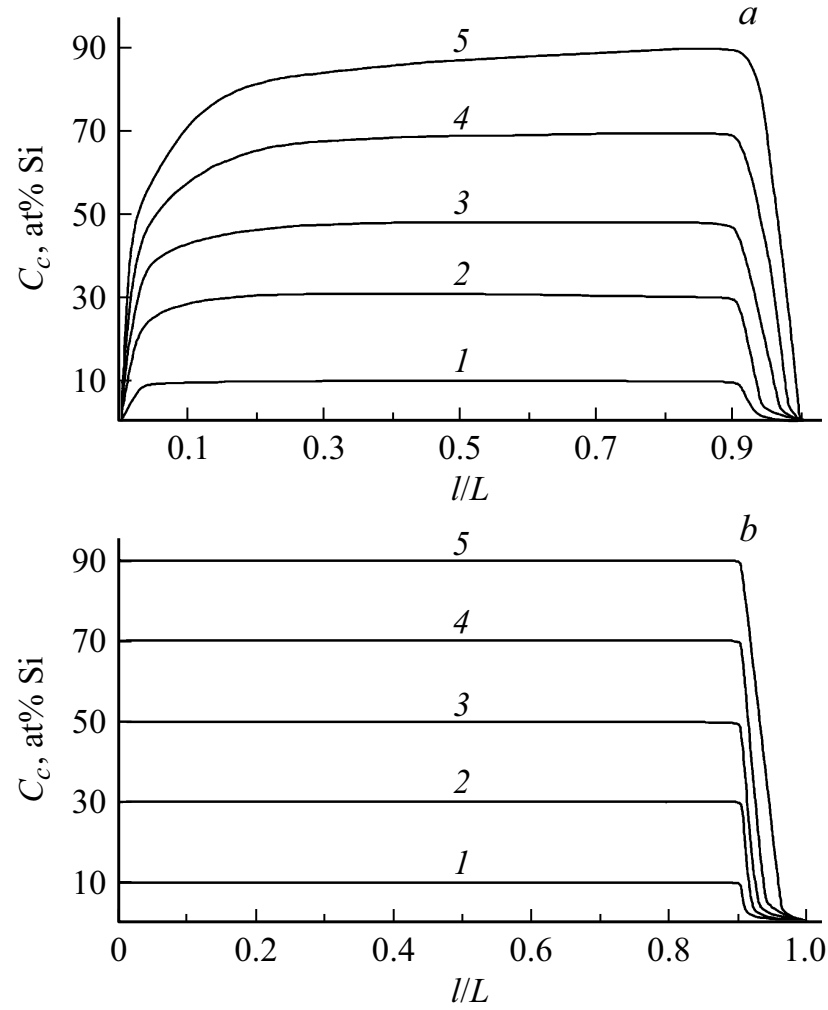

Рис. 2. Расчетные кривые концентрационного распределения $\mathrm{Si}$ по длине кристаллов $\mathrm{Ge}-\mathrm{Si}$, выращенных методом трехкомпонентной зонной плавки с использованием затравок из $\mathrm{Ge}(a)$ и $\mathrm{Si}(b)$ при $Z=0.1 L$ : кривые $1-5$ отвечают значениям $C_{i}=0.1,0.3,0.5,0.7$ и 0.9 соответственно.

Кривые рис. 2, $b$, для случая с Si-затравкой, рассчитаны из уравнений (4) и (7) для участков от затравки до конечной расплавленной зоны и после ее образования соответственно. Как видно, здесь для всех пяти значений $C_{i}$, с момента начала кристаллизации до образования финальной расплавленной зоны идет рост полностью однородных кристаллов с концентрацией кремния, равной соответствующему значению $C_{i}$.

Аксиальное концентрационное распределение кремния в процессе перекристаллизации конечной зоны при использовании затравок как из $\mathrm{Ge}$, так и $\mathrm{Si}$, для рассмотренных случаев, идентично для соответствующих значений $C_{i}$ подпитывающих стержней. На этом участке все кривые демонстрируют крутой спад концентрации кремния по длине кристалла от значения $C_{c} \approx C_{i}$ до $C_{c} \approx 0$.

На рис. 3 представлены расчетные кривые концентрационного распределения кремния вдоль кристаллов

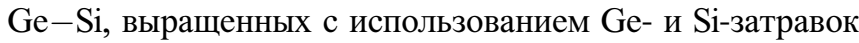
при четырех различных значениях $Z$. Для примера, в расчетах состав подпитывающего слитка принят $C_{i}=0.5$. Как видно из этого рисунка, операционный параметр $Z$ существенно влияет на перераспределение компонентов при зонной перекристаллизации исходного стержня $\mathrm{GeSi}$ заданного состава. При этом длины как однородной, так 

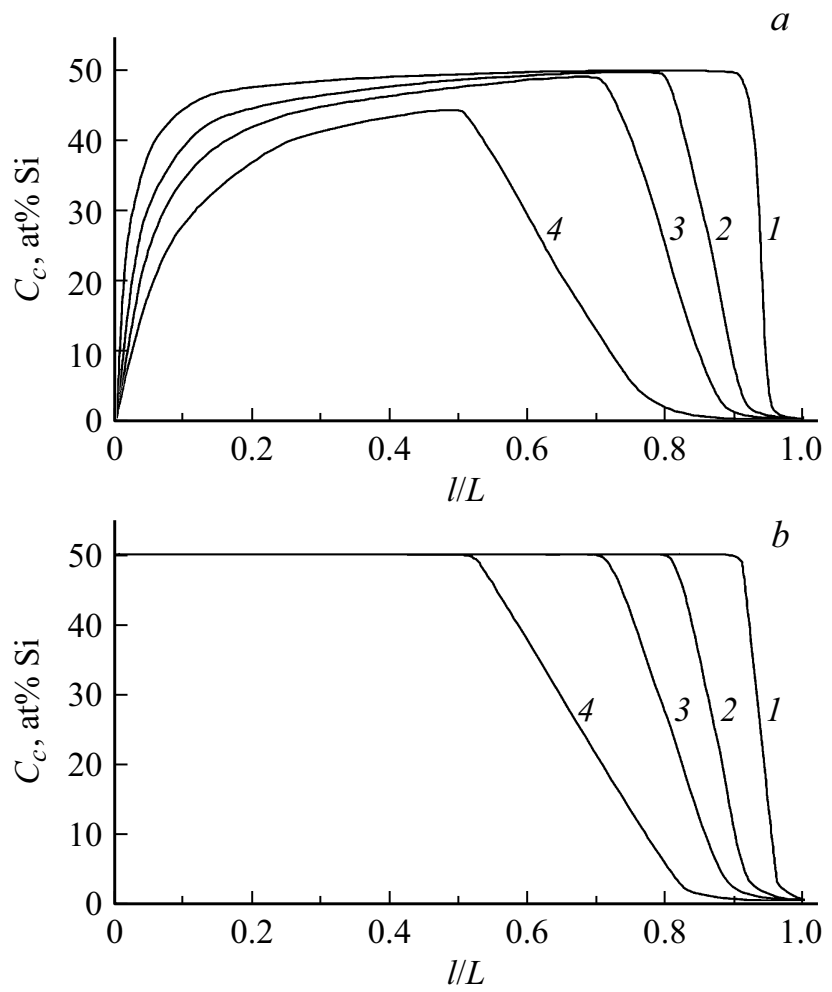

Рис. 3. Концентрационные профили $\mathrm{Si}$ в кристаллах $\mathrm{Ge}-\mathrm{Si}$, выращенных методом трехкомпонентной зонной плавки с использованием затравок из $\mathrm{Ge}(a)$ и $\mathrm{Si}(b)$ при $C_{i}=0.5$ : кривые $1-4$ соответствуют значениям $Z=0.1,0.2,0.3$ и $0.5 L$.

и неоднородной по составу частей кристалла определяются величиной $Z$ расплавленной зоны.

Семейства кривых рис. 2 и 3 наглядно демонстрируют потенциал метода трехкомпонентной зонной плавки для получения монокристаллов $\mathrm{Ge}-\mathrm{Si}$ с требуемым однородным и переменным составами во всем непрерывном ряду твердых растворов. При этом математическое моделирование концентрационных профилей компонентов в кристаллах дает возможность произвести оценку оптимальных технологических параметров $\left(Z, C_{i}\right.$, материал затравки $\mathrm{Ge} / \mathrm{Si}$ ) для получения твердых растворов с конкретно заданными свойствами.

\section{4. Заключение}

Резюмируя вышеизложенное, можно констатировать, что метод трехкомпонентной зонной плавки имеет реальные перспективы для выращивания объемных монокристаллов широкого ряда полупроводниковых твердых растворов с заданным аксиальным концентрационным профилем компонентов.

В заключение вкратце о решении задачи, сформулированной в начале работы, по определению технологических условий и режимов кристаллизации расплава, обеспечивающих монокристалличность твердых растворов. Известно, что рост монокристаллов твердых растворов может иметь место при достаточно малых скоростях кристаллизации расплава. Например, для концентрированных твердых растворов системы $\mathrm{Ge}-\mathrm{Si}$ скорость роста монокристаллов более чем на 2 порядка ниже скорости роста их составных компонентов [17,22]. Связано это с явлением концентрационного переохлаждения расплава вблизи зоны основного фронта кристаллизации. Возникает оно в результате существенной сегрегации компонентов в процессе кристаллизации расплава и является причиной нарушения монокристалличности растущего твердого раствора. Критическая скорость $v_{c}$, выше значения которой происходит поликристаллизация растущего кристалла, может быть оценена с помощью известной формулы Тиллера [17]. Зависит эта скорость от таких параметров, как состав расплава, градиент температуры у фронта кристаллизации, коэффициенты сегрегации компонентов системы и т.д.

Для обеспечения монокристалличности твердых растворов $\mathrm{Ge}-\mathrm{Si}$, выращенных рассмотренным методом, необходимо применение таких технологических режимов, при которых в процессе всего цикла кристаллизации расплава скорость роста кристалла на участках, как с переменным, так и с однородным составами (рис. 2 и 3 ), не будет превышать соответствующие значения $v_{c}$.

\section{Конфликт интересов}

Автор заявляет об отсутствии конфликта интересов.

\section{Список литературы}

[1] J. Schilz, V.N. Romanenko. J. Mater. Sci.: Mater. Electr., 6, 265 (1995).

[2] A. Barz, P. Dold, U. Kerat, S. Recha, K.W. Benz, M. Franz, K. Pressel. J. Vac. Sci. Technol. B, 16, 1627 (1998).

[3] C. Marin, A.G. Ostrogorsky. J. Cryst. Growth, 211, 378 (2000).

[4] S. Adachi. J. Cryst. Growth, 280, 372 (2005).

[5] T.A. Campbell, M. Schweizer, P. Dold, A Cröll, K.W Benz. J. Cryst. Growth, 226, 231 (2001).

[6] Г.Х. Аждаров, 3.М. Зейналов, 3.А. Агамалиев, А.И. Кязимова. Кристаллография, 55, 763 (2010).

[7] A. Varilci, T. Kucukomeroglu, G.Kh. Azhdarov. Chin. J. Phys., 41, 79 (2003).

[8] G.Kh. Azhdarov, T. Kucukomeroglu, A. Varilci, M. Altunbaş, A. Kobya, P.G. Azhdarov. J. Cryst. Growth, 226, 437 (2001).

[9] N.V. Abrosimov, S.N. Rossolenko, W. Thieme, A. Gerhardt, W. Schroder. J. Cryst. Growth, 174, 182 (1997).

[10] I. Yonenaga. J. Cryst. Growth, 275, 91 (2005).

[11] V.K. Kyazimova, Z.M. Zeynalov, Z.M. Zakhrabekova, G.Kh. Azhdarov. Crystallography Reports, 51 (1), 192 (2006).

[12] Г.Х. Аждаров, 3.М. Зейналов, Л.А. Гусейнли. Кристаллография, 54 (1), 137 (2009).

[13] I. Yonenaga, T. Ayuzava. J. Cryst. Growth, 297, 14 (2006).

[14] P. Dold, A. Barz, S. Recha, K. Presse, M. Franzb, K.W. Benza. J. Cryst. Growth, 192 (1-2), 125 (1998).

[15] 3.М. Захрабекова, 3.М. Зейналов, В.К. Кязимова, Г.Х. Аждаров. Неорг. матер., 43 (1), 5 (2007). 
[16] S. Bok-Cheol, K Kwang, L. Hong-Woo. J. Cryst. Growth, 290, 665 (2006)

[17] Г.Х. Аждаров, З.А. Агамалиев, Э.М. Исламзаде. Кристаллография, 59, 489 (2014).

[18] Z.A. Agamaliyev, E.M. Islamzade, G.Kh. Azhdarov. Crystallography Reports, 61, 327 (2016).

[19] M. Yildiz, S. Dost, B. Lent. J. Cryst. Growth, 280, 151 (2005).

[20] K. Nakajima, T. Kusunoki, Y. Azuma, N. Usami, K. Fujiwara, T. Ujihara, G. Sazaki, T. Shishido, J. Cryst. Growth, 240, 373 (2002).

[21] В.М. Глазов, В.С. Земсков. Физико-химические основы легирования полупроводников (М., Наука, 1967).

[22] I. Yonenaga. J. Cryst. Growth, 198/199, 404 (1999).

[23] П.Г. Аждаров, Н.А. Агаев. Неорг. матер., 35, 763 (1999).

[24] I. Kostylev, J.K. Woodaste, Y.P. Lee, P. Klages, D. Labrie, J. Cryst. Growth, 377, 147 (2013).

Редактор А.Н. Смирнов

\section{Three-component zone melting method: Modeling of components distribution concentration in single crystals of Ge-Si solid solution}

Z.A. Aghamaliyev ${ }^{1,2}$

${ }^{1}$ Baku State University, Az-1148 Baku, Azerbaijan

${ }^{2}$ Institute of Physics,

National Academy of Sciences of Azerbaijan,

Az-1143 Baku, Azerbaijan

Abstract The concept and fundamentals of the three-component zone melting method for growing single crystals of semiconductor solid solutions using seeds from constituent components is given. Using Pfann approximation,were calculated the concentration profiles of the components along the crystal axis in $\mathrm{Ge}-\mathrm{Si}$ single crystals grown at different values of the operating parameters, such as the length of the molten zone and the composition of the initial macrohomogeneous rods of solid solutions. An analysis of the results yields the optimal technological parameters and conditions for growing single crystals with specified homogeneous and gradient compositions in the entire continuous series of $\mathrm{Ge}-\mathrm{Si}$ solid solutions. It is shown that the three-component zone melting method is promising for growing single crystals of semiconductor solid solutions. 